\title{
Entre fidélité et libertés interprétatives : l'adaptation numérique à la lumière du programme Collecta
}

Between Fidelity and Interpretative Freedom: Digital Adaptation in the Light of the Collecta Research Programme

\section{Sophie Fétro}

\section{OpenEdition \\ Journals}

Édition électronique

URL : https://journals.openedition.org/revuehn/437

DOI : $10.4000 /$ revuehn. 437

ISSN : 2736-2337

Éditeur

Humanistica

\section{Référence électronique}

Sophie Fétro, «Entre fidélité et libertés interprétatives : l'adaptation numérique à la lumière du

programme Collecta », Humanités numériques [En ligne], 2 | 2020, mis en ligne le 01 juin 2020, consulté le 16 juillet 2021. URL : http://journals.openedition.org/revuehn/437 ; DOI : https://doi.org/10.4000/ revuehn. 437

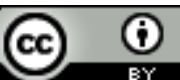

Les contenus de la revue Humanités numériques sont mis à disposition selon les termes de la Licence Creative Commons Attribution 4.0 International. 


\section{humanités \\ numériques}

\section{Entre fidélité et libertés interprétatives : l'adaptation numérique à la lumière du programme Collecta \\ Between Fidelity and Interpretative Freedom: Digital Adaptation in the Light of the Collecta Research Programme}

\section{Sophie Fétro}

\section{Résumés}

Comment opérer le passage d'une collection historique de papier à sa version informatique ? C'est en prenant appui sur le programme de recherche "Collecta. Archive numérique de la collection Gaignières (16421715) ", fondé sur la rencontre de l'histoire de l'art, de l'informatique et du design, que cet article propose d'étudier les processus d'adaptation qui ont lieu lors de ce changement d'état. Pour étudier ce phénomène, deux autres pratiques artistiques impliquant des techniques de reproduction et de diffusion de masse - la gravure et le cinéma - seront convoquées car elles impliquent, du fait même des appareils et de leurs modalités de fonctionnement, des processus d'adaptation. Loin de les confondre, il s'agit plutôt de repérer ce qui s'opère lors de ce transfert de support (du papier à l'écran), de déceler les potentialités des techniques numériques en termes de lecture, de modalités classificatoires et d'accès à la connaissance, mais aussi de découvrir ce qu'André Bazin appelle, à propos du cinéma, leurs "structures esthétiques " et leurs " moyens propres".

How to make the transition from an historical collection of paper to its digital version? Drawing from the research program "Collecta. Digital Archive of the Gaignières Collection (1642-1715)", based on the interaction of art history, computer science and design, this article proposes to study the adaptation processes that take place during such a transfer. To study this phenomenon, two other artistic practices and techniques of 
reproduction and mass diffusion - engraving and cinema - will be discussed because their devices and operating methods entail adaptation processes. Far from confusing them, the aim is to identify what is happening during this change of media (from paper to screen), to find out the potentialities of digital techniques in terms of reading, classification and access to knowledge, and also to discover what André Bazin calls, with regard to cinema, their "aesthetic structures" and their "specific means of action”.

\section{Entrées d'index}

MOTS-CLÉS : design, histoire, adaptation, cinéma, collection, gravure, interface, multimédia, numérisation, Web

KEYWORDS: design, history, adaptation, cinema, collection, engraving, interface, multimedia, digitisation, Web

Né de la rencontre entre l'historienne de l'art Anne Ritz-Guilbert et Sophie Fétro, maître de conférences spécialisée en design, le programme de recherche Collecta qu'elles ont co-dirigé pendant plus de deux ans (de juin 2014 à juin 2016) a été porté par l'École du Louvre, en partenariat avec l'université Paris 1 Panthéon-Sorbonne et financé par la COMUE HéSam dans le cadre des projets Synergie. Ayant pour but de fédérer des équipes pluridisciplinaires, le projet a pu se concrétiser grâce à une solide équipe de spécialistes, de futurs chercheurs, de chercheurs confirmés et d'informaticiens, aux compétences complémentaires ${ }^{1}$. Il s'est concrétisé à travers la création d'une base de données et d'un site Web associé : collecta.fr ${ }^{2}$, accessible en ligne et hébergé par l'IRHT. L'enjeu de ce projet a consisté, avec les moyens du numérique, à « restituer les classements et processus d'élaboration d'une collection historique du XVII $^{\mathrm{e}}$ siècle ", celle du collectionneur et antiquaire François-Roger de Gaignières. Ce dernier " passionné par l'histoire de la noblesse et de la monarchie française ", a sillonné la moitié nord de la France avec son dessinateur Louis Boudan et son copiste Barthélemy Rémy, « dépouillant pendant presque un demi-siècle, les chartriers des châteaux, des abbayes et des chapitres, faisant copier et relever des centaines de milliers d'actes, de titres et de monuments (tombeaux, épitaphes, plaques de fondation, vitraux, sceaux, tapisseries, etc.) ${ }^{3}$ ". Il laisse derrière lui « une collection documentaire exceptionnelle sur les époques médiévale et moderne ${ }^{4}$ " encore largement utilisée aujourd'hui. Cette collection composée à la fois d'écrits, de dessins et de quelques objets, dont l'inventaire de 1711 recense plus de 5300 items, livre un inventaire remarquable de monuments historiques (préfigurant les politiques patrimoniale et de conservation des bâtiments historiques), aussi bien qu'un témoignage de la sculpture médiévale et de la mode du Moyen Âge au XvII ${ }^{\mathrm{e}}$ siècle, participant d'une culture visuelle hors du commun qui justifie dans une large mesure l'existence de ce programme de recherche. Quatre objectifs ont motivé le travail de recherche :

- restituer et documenter scientifiquement la collection Gaignières 
- inventer un mode de représentation permettant à chacun de visualiser la collection dans son ensemble pour appréhender son volume, son classement et accéder à chaque pièce dans son contexte

- considérer la fortune critique et artistique de la collection à travers ses copies et usages ultérieurs

- mettre en mouvement la collection, réassembler les documents selon d'autres combinaisons intellectuelles et visuelles, susciter ou autoriser de nouveaux questionnements ${ }^{5}$

\section{La question de l'adaptation}

C'est en partant du constat d'un changement de matérialité qu'induisait le transfert de la collection de papier de Fr.-R. de Gaignières vers sa consultation numérique que la question de l'adaptation s'est fait jour, induisant des modifications dans la consultation des planches et des documents, leur perception ainsi que leur accès. Comment adapter cette collection historique au regard des possibilités offertes par le numérique mais aussi de ses contraintes, sans perdre, ni son identité, ni la singularité de sa logique classificatoire, tout en apportant quelque chose de neuf ? Afin de conceptualiser cette problématique de création, l'étude d'autres champs artistiques peut permettre de comprendre comment la question de l'adaptation a pu être traitée et a pu se concrétiser, dans le but de synthétiser le positionnement intellectuel et créatif qui a été le nôtre. C'est en référence à des champs et pratiques a priori éloignés des humanités numériques - la gravure et le cinéma -, au regard de leur histoire, de leurs modalités techniques et de leurs usages, que je propose d'appréhender cette question de l'adaptation numérique afin d'identifier les logiques opératoires qu'implique un projet en humanités numériques comme celui consacré à la collection de Fr.-R. de Gaignières. C'est en partant de l'hypothèse que les humanités numériques, au regard des processus de transfert qu'elles impliquent (numérisation des documents, classification des données, modalités d'affichage et de présentation des documents, etc.), peuvent être pensées comme adaptatrices, c'est-à-dire comme un domaine de pensée et d'organisation du savoir qui procède à des opérations d'adaptation, que cet article entend analyser l'expérience Collecta. Les comparaisons non synchroniques ici proposées ont pour but de montrer que les techniques adaptantes auxquelles recourent les humanités numériques recoupent dans la culture des problématiques plus anciennes, conduisant à des transferts de présentation ; et qu'à travers elles se posent des questions à la fois techniques, esthétiques, d'accès à des contenus, d'usage, de diffusion et de réception.

\section{Médiations numériques dans le champ de l'histoire de l'art}

Aujourd'hui les campagnes de numérisation sont légion au sein des lieux d'archives, des bibliothèques et des fonds patrimoniaux (collections, musées). Des sommes importantes et nécessaires sont allouées aux procédures de numérisation, impliquant l'achat de matériels techniquement sophistiqués et coûteux, l'emploi et la spécification de person- 
nels qualifiés dédiés à cette tâche, des espaces spécifiques et du temps pour réaliser ces opérations. Pour autant que ces démarches sont importantes et constituent un travail considérable au sein des organismes en charge de cette mission, ce changement d'état ne fait pas souvent l'objet d'une réflexion approfondie sur le plan notamment de l'usage et de la forme, autrement dit sur le plan de leur design. Les campagnes de numérisation sont généralement faites de façon systématique, sans que soient véritablement distingués les documents traités et scannés. Or, bien que la nature d'une enluminure, d'un manuscrit, d'un petit objet ou d'un tableau diffèrent strictement, souvent les procédures de numérisation, les technologies employées et les présentations de l'information sont identiques : un même format de vignettes ne prenant pas en considération les échelles des objets à travers des mosaïques d'images indifférenciées, des modalités de consultation et d'interrogation similaires. Ces campagnes, qui constituent une première étape dans le processus de traitement des données, ne sauraient cependant suffire à produire des interfaces pertinentes au regard de ce qu'elles abritent. Dans ce contexte, un projet de recherche en humanités numériques impliquant une approche par le design peut avoir du sens afin d'interroger une situation spécifique, d'envisager les meilleurs choix à effectuer quant à la présentation des objets traités et à la façon d'y avoir accès. Une telle démarche peut précisément être l'occasion de penser ce passage entre deux réalités (analogique et numérique), d'avoir le temps de le faire, de regrouper des équipes pluridisciplinaires (constituée d'historiens, d'historiens de l'art, de designers et d'informaticiens, de développeurs, de spécialistes du Web sémantique, etc.), et de restituer ainsi les conclusions des chercheurs de façon publique. Ces dernières années, plusieurs projets scientifiques relevant du champ de l'histoire de l'art, de l'archéologie et des humanités numériques ont vu le jour. Citons, outre $\operatorname{Collecta}^{6}: \operatorname{ENCCRE}^{7}$, projet d'édition numérique collaborative et critique de l'Encyclopédie de Diderot et d'Alembert qui « met pour la première fois à disposition de tous les connaissances des chercheurs d'hier et d'aujourd'hui sur l'Encyclopédie, en s'appuyant sur un exemplaire original et complet de l'ouvrage, conservé à la bibliothèque Mazarine, intégralement numérisé pour l'occasion "; Arachné $^{8}$ ( "Un regard critique sur l'histoire de la tapisserie ", mené par Pascal-François Bertrand et Audrey Nassieu Maupas), programme de recherche soutenu par l'ANR et la région Aquitaine qui vise à replacer la tapisserie au sein de l'histoire de la création artistique ; Guides de Paris ${ }^{9}$ (projet inscrit dans le cadre du Labex « Les passés dans le présent " et mené par Emmanuel Château et Marianne CojannotLe Blanc), qui " consiste à produire une édition critique électronique d'un corpus de guides de Paris des XVII ${ }^{\mathrm{e}}$ et XVIII ${ }^{\mathrm{e}}$ siècles "; E-Stampages ${ }^{10}$ (mené par Michèle Brunet et Adeline Levivier - UMR 5189 HiSoMA, Histoire et sources des mondes antiques - et l'École française d'Athènes), qui " réunit dans une publication numérique deux des plus riches collections d'estampages d'inscriptions grecques, celle de l'École française d'Athènes et celle du laboratoire HiSoMA "; EAT Datascape ${ }^{11}$ (The Experiments in Art and Technology), mené par Christophe Leclercq, Paul Girard, Patrick Browne et Daniele Guido (Médialab de Sciences Po), lesquels proposent une exploration des archives numériques d'un projet de l'artiste américain Robert Rauschenberg (cofondateur de EAT), pour faciliter la collaboration entre artiste, ingénieur et industrie. Ces projets à caractère scientifique restent malgré tout assez peu nombreux au regard de la 
masse de documents existants. Ils se situent en tout cas à la marge dans les processus de numérisation des fonds documentaires et des collections historiques dans le champ de l'histoire de l'art, ce qui conduit à dissocier, d'un côté, les bases de données qui donnent la priorité à "l'interrogeabilité » des contenus et, de l'autre, les sites Web plus spécifiques, proposant une expérience utilisateur inédite et pour lesquels l'interface graphique, les modalités d'affichage, de consultation et d'exploration des données font l'objet d'un traitement particulier (plus ou moins approfondi néanmoins) dans le but d'entrer en résonance avec leur objet.

\section{Respecter la source}

Par un effet métonymique ou par facilité, il pourrait être tentant d'assimiler une base de données à la collection elle-même. Or, un site Internet ou une base de données ne sont jamais les reproductions exactes d'une collection historique, car, de fait, les modalités d'archivage et d'affichage informatiques diffèrent des documents papier. Un processus d'interprétation des données a lieu, qu'il soit humain ou informatique : humain, parce que l'historien va opérer une interprétation des informations historiques ; informatique, parce qu'un encodage est indispensable pour intégrer les données, soit de façon automatisée, soit de façon manuelle afin de les rendre interopérables et consultables. Ce processus interprétatif, inévitable dans ce passage du papier au numérique, peut présenter des risques d'erreur, de surinterprétation, de déformation et d'altération, qui pourraient conduire à trahir la source, un peu comme le bouche-à-oreille où le passage de relais et la multiplication des intermédiaires peuvent dénaturer le propos initial. C'est en effet le "risque " nécessaire à prendre lorsqu'un projet en humanités numériques est entrepris. Il peut toutefois être grandement atténué si des allers-retours et échanges répétés ont lieu entre les différents acteurs du projet et si des tests sont fréquemment réalisés afin de vérifier la fiabilité des informations et la cohérence de la base de données. Pour autant que ce risque existe, l'écart qui, de fait, sépare la source de la version numérique n'est pas nécessairement l'expression d'une trahison " perverse " à l'encontre de la source. Cela peut renvoyer davantage à la " nécessaire trahison du traducteur " (Pédauque 2006) lors du passage d'une langue à une autre. De la même façon que "le point aveugle des approches technologiques de la traduction concerne la compréhension de ce qu'est un texte et de la manière dynamique dont se construit son sens ", lécueil de la traduction numérique d'une collection en langage informatique pourrait être d'occulter la spécificité de cette collection et la tentation d'une automatisation de l'ensemble des procédures. Le travail interprétatif qu'implique le passage d'une collection historique vers son existence numérique, loin de relever d'une infidélité dommageable pour elle, peut s'avérer bénéfique dans la découverte de cette collection, de sa lecture critique, de sa compréhension et de sa diffusion. Toujours selon Roger T. Pédauque : "Le traducteur professionnel résout les problèmes locaux en fonction d'une appréhension globale du texte, en combinant les différents niveaux du texte. Il s'ensuit que le texte traduit est toujours une transformation de l'original. Contrairement aux idées reçues, la trahison du traducteur résulte d'une fidélité captive, d'un assujettissement aux mots 
de l'original. Le traducteur ne raisonne pas en termes de traduction littérale mais d'équivalence [...]. La part de création que suppose toute traduction passe par des prises de décision qui ne relèvent pas d'une axiologie explicite ", mais, pourrait-on dire, de processus d'interprétation. Assurément, les procédures informatiques, les appareils, l'encodage des documents constituent des " opérateurs transformationnels ${ }^{12}$ ", notamment au moment de la numérisation des documents, qui agissent sur l'état des reproductions et leur perception. D'autres facteurs ensuite jouent dans la "variance ${ }^{13}$ " d'un document, comme le choix du format d'une image agissant sur ses "propriétés formelles » et la qualité de son affichage à l'écran (induisant une plus ou moins grande pixellisation). Mais il faut distinguer les procédures de dégradation ou d'altération des documents non souhaitées, inhérentes aux procédures elles-mêmes de captation et d'encodage pouvant engendrer une perte qualitative, des processus de transformation volontaires qui engagent un processus créatif et sur lesquels il est possible d'agir en opérant des choix en termes de structure de l'information et de présentation des documents. Bien que des limites existent, comme la résolution des images, des avantages peuvent être retirés de ces processus transformationnels qu'implique l'informatique. Assurément, le site Web collecta.fr est une façon d'appareiller la collection Gaignières et de modifier ses modes de lecture, de consultation et d'interrogation. Cependant, l'enjeu pour Collecta a consisté à se tenir au plus proche de la collection Gaignières. C'est la raison pour laquelle l'organisation des documents au sein de la base de données et du site a respecté scrupuleusement l'inventaire de 1711 (figure 1) mis en place au moment de la cession de la collection au roi de France afin d'en dresser un état des lieux complet. 


\section{COLLECTA

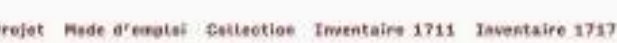

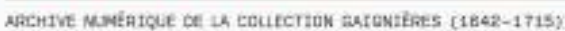

Inventaire de 1711

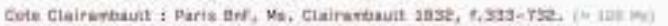

\section{Teat dipliter}

MANUSCRITS

+ Bibles, tivres ecclesiastinues

+ Droit canon et civit

Paitosophie, solitique, mathenatique, arts, ete.

+ Medecine

Geogrephie, histeire univerastis

4 Histoires neciesisstiques, papes, eavdinaus, atc., srehovesques, ouesques,

shbet, privurs, retielewe, ote.

+ Histoire generate de France

+ Recueits conoernant ('histoire des orovinees de france

+ Histoire etrangere

+ Histoire des ordees de chevaleries

+ Histoires particutieres of vies

+ Histoire des familles

+ anitess Lottres

+ Miscellanei incerti

PORTEFEUILS ET VOLUMES [REMPLIS D'ESTAMPES, PORTRAITS,

MODES, DESSEINS, TOMBEAUX, ETC.] QUI SONT DANS LA

GALLERIE, ILS SONT TOUS GRAND IN-FOLIO.

+ [Portralts Eravés]

+ Geographie, topouraphis

+ Nodes

t Tombeaux et autres mofumens

+ JETTONS

+ ADDITIONS

+ PAQUETS NON RELIEZ

IMPRIME 2

+ Livres de devetion et de matieres de theotogie

+ Oroit civit et canan

+ Pollosophie, politique, arts, ete.

+ Medeoline

Histoire universelte

Histore sacrie et ecelesiastique

+ Histoire generats de Franes

t Vies cet cloges.

+ Histaires particutieres des provinces de France

+ Histoiro etranare

+ Genealogies ot mistoires des famittes

+ Ordes de chevalerio.

+ Belles lettres

+ Habits, mbdes, atc.

+ Misectiansi

\section{TABLEAUX ET PORTRAITS}

+ Ceux du catinet apres La chambre de tarade. Th ont teus des bordure

ostare:

+ Cuux du srand cabinat arant ta gallerie

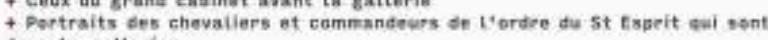

enns in gallerie

SECONDE ADDITION FAITE À L'INVENTAIRE DU CABINET DE MR.

DE GAIGNIERES EN 1715

+ Manuscrits

+ Geographie, topoeraphie, portraits, tombeaux, modes, festes, etc.

Jettons, medailles et monnoyes, pourcelafoe, ete.

+ Aoditions d'ingrimez: 1710

+ Tableair et portraits

touige Mantieas ikgaies Santact Danrasian

Page correspondant à l'inventaire de 1711 à partir duquel le site et la base de données se sont structurés. On y retrouve les 5 entrées du modèle par points : « Manuscrits », « Portefeuilles », «Imprimés ", « Tableaux ", « Objets ».

Programme de recherche Collecta, site Web : https://www.collecta.fr

Cinq grands ensembles avaient alors été distingués : "Manuscrits", " Portefeuilles de dessins et d'estampes ", «Imprimés ", " Portraits et tableaux » et, enfin, « Monnaies, médailles et jetons » (Paris, BNF, ms. Clairambault 1032), ce que nous avons appelé «Objets ». En outre, l'une des particularités de la collection Gaignières repose sur ses portefeuilles re- 
groupant des documents de nature variée : vues topographiques, représentations de monuments et de bâtiments, vitraux, tombeaux, costumes, textes (mêlant originaux et copies) (figure 2).

FIGURE 2. APERÇU DES TYPES DE DOCUMENTS

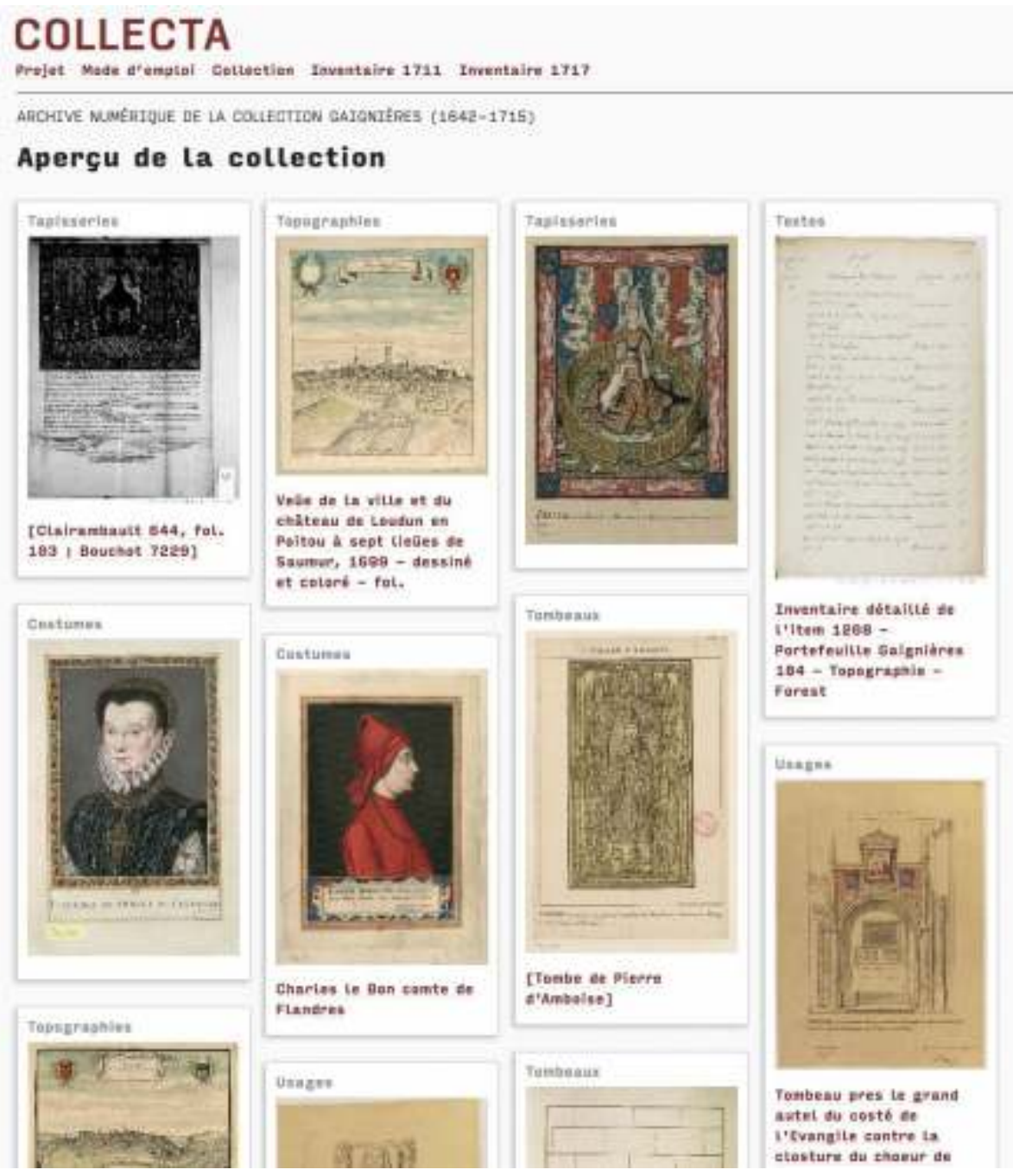

Visualisation du contenu de la collection à travers un aperçu des différents types de documents que l'on peut y trouver : tapisseries, topographies, textes, costumes, tombeaux, vitraux, usages ; traduisant l'esprit du « musée de papiers » qu'était la collection Gaignières. À chaque ouverture du site, un nouvel affichage se met en place de façon aléatoire, offrant de possibles entrées dans la collection, sans cesse renouvelées.

Programme de recherche Collecta, site Web : https://www.collecta.fr

Souhaitant rester fidèle à l'idée de « musée de papiers » que Gaignières expose de son vivant (du moins en partie) à ses visiteurs, nous avons proposé une présentation des documents sous forme de planches «volantes ». Par ailleurs il procède lui-même à différents procédés adaptatifs. Le cas des modes est à cet égard spécifique et typique d'un processus qu'il met en place, proposant une interprétation dessinée en pied d'effigies funéraires, rendues comme vivantes par ce procédé, et constitutif d'une documentation exemplaire pour l'histoire du costume. L'un des grands «miracles » des liens hypertexte a été de pouvoir reconstituer les portefeuilles initiaux (figures 3 et 4 ). 
17 Veüe de l'église cathedralle de St Pierre de Poitiers du coté de la grande porte - dessiné et coloré - fol.

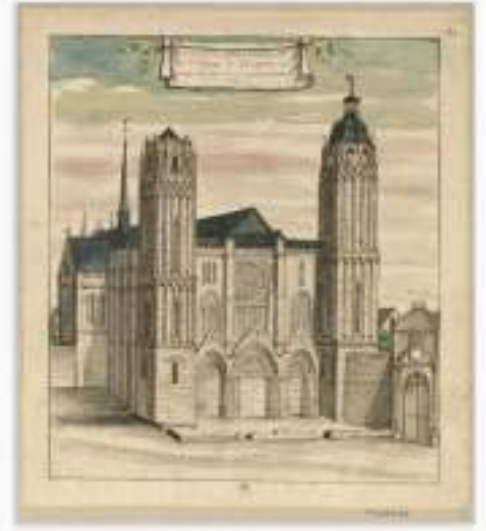

Irii se Paris I BNF (Defartement des Estakpes ot de to

phetegraphic:

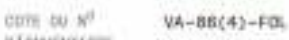

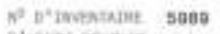

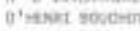

motide te

1802

thrivent

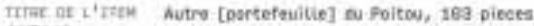

(1711)

C' maser

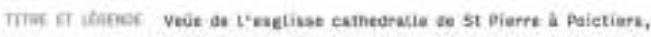
dassing do pasit on ta grande parts

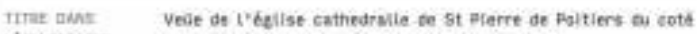

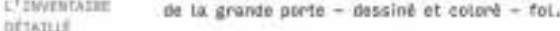

Vue de la façade de la cathédrale de Poitiers, dans le portefeuille du Poitou.

Programme de recherche Collecta, site Web : https://www.collecta.fr 
Secenstitution to meush e'arisint
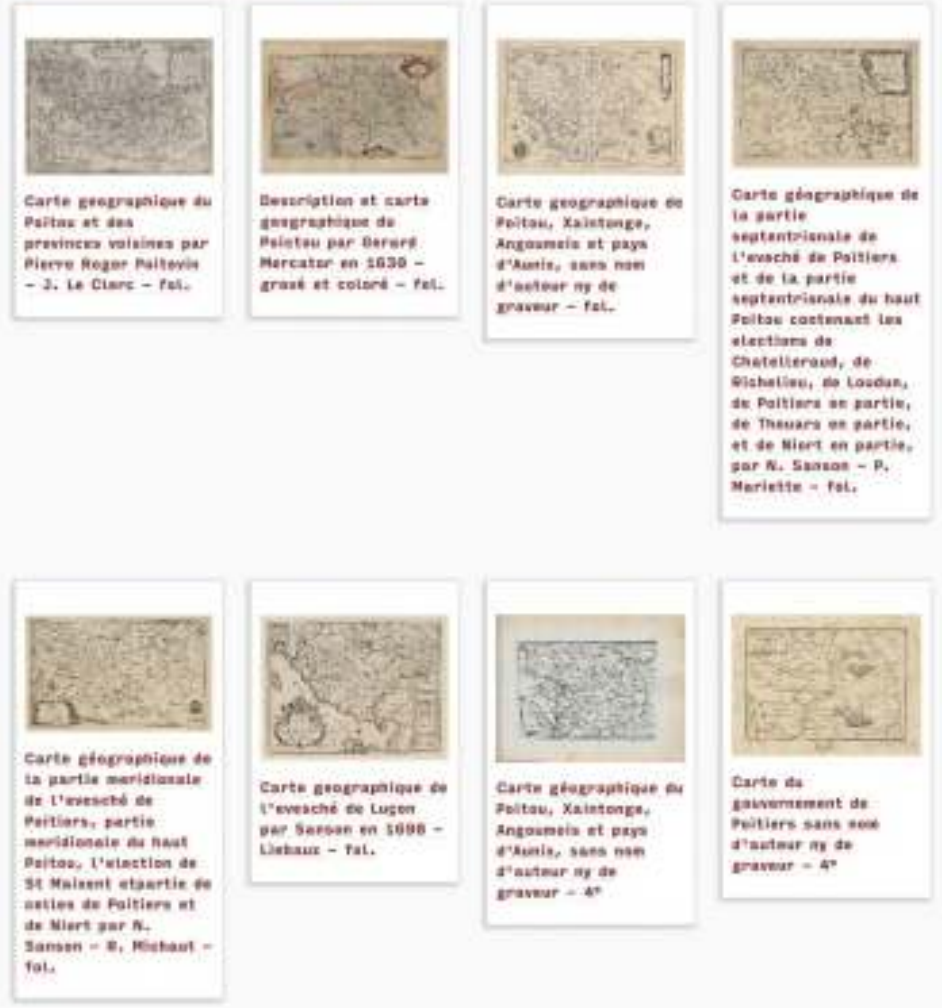

ta sertic

teatentoisnale ate

1.evesche on Paisiers

it de la partie

goltas coetenast tes

atectian de

Gietelieraed, at

ciaholive, it Losouk,

se thewars an partie.

thas miert en partie.

Meriette - tot.
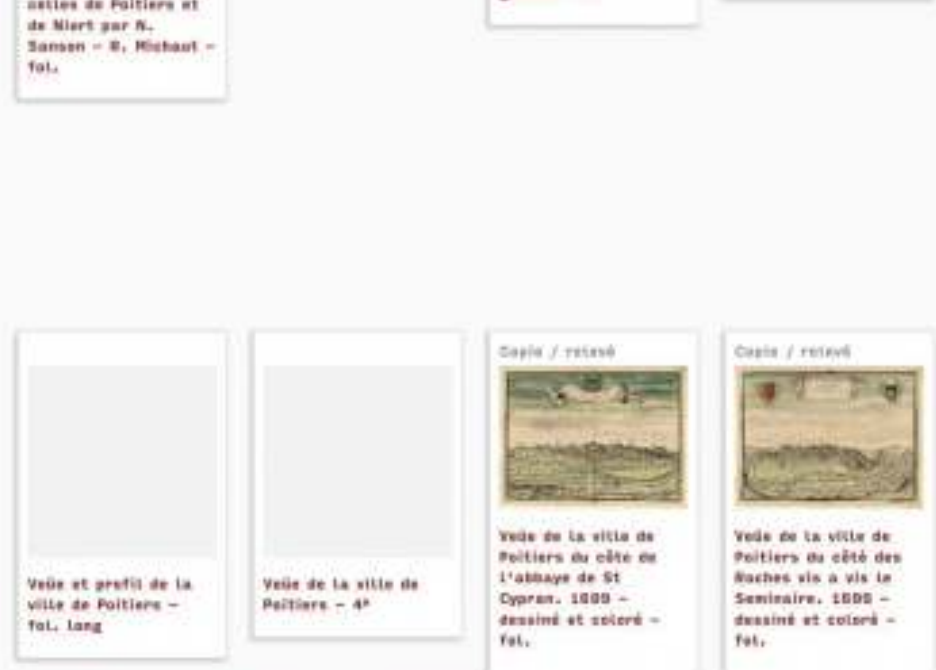

weas to ta vitie as peitiers of elite $\mathrm{a}$ 1'atiane de st tratint ot asierd rat.

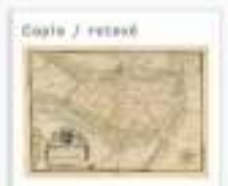

pian ae is vilie de. asitiers - dessies at. metiert - tot

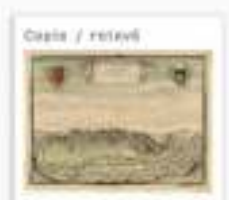

vede tee ix veliz de poitiens as eeth de Saninairs. tems tratint ot coiserd tot.

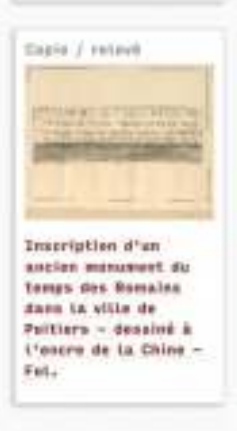

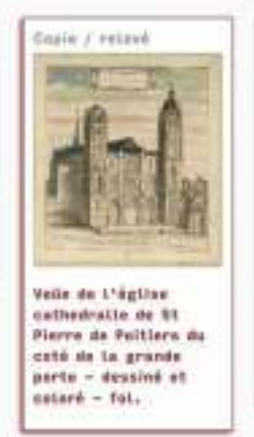

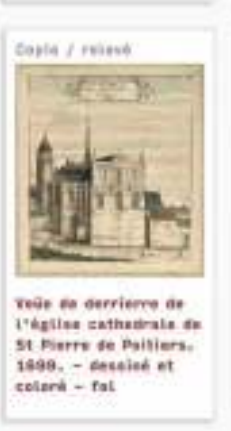

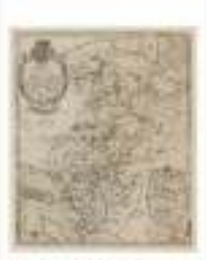

carts chaeranatiage of pass t'hunia ot partie is senectassste do is. Fachotie et partis do teticn as pritiviv Co Faniesay.

Rochetie et partie to cetin en remteray at Co
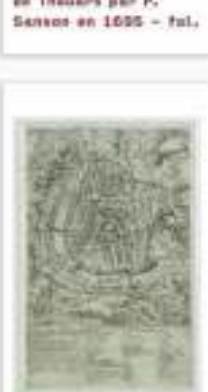

Phan de ia ville te Doitiers asaiegét en 1503 war it rey te
Mavarts, 64 griest to Dende, iramirnt te colievs et it comte ad Mantela, thets an magutels,

extrendie far te dut Ce bulse $n t$ io enats Ec Luats ail as itoit

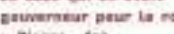
- pierre - thet.

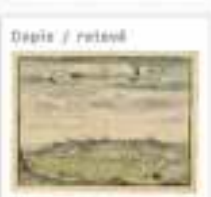

Vele de tu vilia as

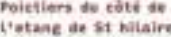
$1 \mathrm{kns}$ - thesaind atart $\rightarrow$ tois

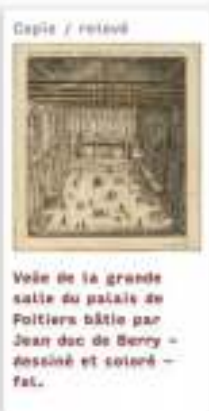

Il est possible de reconstituer le portefeuille d'origine dans l'ordre initial de classement des planches, ici un extrait du portefeuille consacré au Poitou.

Programme de recherche Collecta, site Web : https://www.collecta.fr

Alors qu'aujourd'hui la collection est éclatée en divers lieux de conservation dont les deux principaux sont la $\mathrm{BNF}^{14}$ et la Bodléienne à Oxford, et tandis que certaines pièces ont été perdues, il est possible grâce au référencement et à la structure des données, aux liens hypertexte, d'aller 
chercher dans la base de données tous les documents appartenant à un même portefeuille et de les présenter visuellement sous forme de vignettes conformément à l'ordre qu'avait imaginé Gaignières, ce qui s'avère impossible à faire physiquement depuis la dispersion de la collection Gaignières après sa mort en 1715. Les pages blanches qu'avaient laissées Gaignières pour accueillir des documents à venir ont été respectées, tandis que les documents manquants sont signifiés visuellement par des hachures grises. La puissance de l'outil informatique et l'automatisation de la recherche rendent désormais possibles de telles reconstitutions "virtuelles », ce qui est extrêmement précieux pour les historiens dans la compréhension et l'analyse des documents et de cette collection.

\section{Un parallèle avec l'adaptation au cinéma}

Pour comprendre la nature du processus interprétatif qu'implique la démarche de création d'une base de données et d'un site Web associé dédiés à une collection historique, un parallèle peut être fait avec le cinéma, en particulier avec l'adaptation cinématographique qui se réfère à la littérature ou au théâtre. Le théoricien et critique de cinéma André Bazin, dans son essai Pour un cinéma impur. Défense de l'adaptation (Bazin 1987 [1952]), défend un certain type d'adaptation cinématographique. Pour lui, les bonnes adaptations sont loin d'être seulement des transpositions à l'identique d'une œuvre littéraire. Elles s'avèrent, selon lui, beaucoup plus exigeantes qu'un film construit de toute part, car du talent est nécessaire pour que l'adaptation ne soit pas la réplique à la lettre du roman ou de la pièce de théâtre et ne s'en tienne pas uniquement à la restitution d'un scénario. Pour A. Bazin, la bonne adaptation est celle qui cherche à être fidèle - " Dans le domaine du langage et du style, la création cinématographique est directement proportionnelle à la fidélité ", dit-il (p. 95) - et cette fidélité n'est pas exempte d'invention. Il indique : «Les différences de structures esthétiques rendent plus délicate encore la recherche des équivalences, elles requièrent d'autant plus d'invention et d'imagination de la part du cinéaste qui prétend réellement à la ressemblance. " Ainsi, plus le cinéaste veut être fidèle à la source, plus il cherche à être ressemblant et respecter l'œuvre de référence, plus il lui faut être créatif, car être fidèle au roman implique un travail spécifique qui consiste à faire passer quelque chose du roman avec les moyens du cinéma. Pour que l'adaptation soit réussie, il s'avère donc nécessaire selon lui d'être fidèle à l'esprit du roman : non se contenter seulement d'être fidèle à l'histoire, mais prendre ses distances avec l'œuvre de référence, ne pas vouloir tout dire ou tout montrer, ne pas illustrer l'œuvre littéraire mais l'interpréter : "Pour les mêmes raisons qui font que la traduction mot à mot ne vaut rien, que la traduction trop libre nous paraît condamnable, la bonne adaptation doit parvenir à restituer l'essentiel de la lettre et de l'esprit. " Dans le cas des humanités numériques et de l'adaptation numérique d'un fonds historique, il convient également de chercher à transposer la « lettre » et « l'esprit » de la source, autrement dit, d'entrer en résonance avec la nature même du fonds documentaire et avec le projet qui a été à la naissance de cette collection. A. Bazin ajoute : "Il est faux de se présenter la fidélité comme une servitude nécessairement négative à des lois étrangères. " Non seulement cette « servitude " à l'égard de la source peut être positive mais elle ne dépend pas 
de lois extérieures à l'adaptation. Elle répond précisément à des lois qui lui sont spécifiques, directement liées aux moyens employés. De la même façon que l'adaptation cinématographique constitue un vrai travail et défi créatif, l'adaptation numérique implique une recherche et de la créativité. " Adapter, enfin, n’est plus trahir mais respecter » (p. 99). Il est ici intéressant de penser le respect et la fidélité à la source comme moteur créatif, non pas comme une contrainte qui fige ou qui conduit à répéter ce qui existe déjà, mais comme quelque chose qui stimule l'esprit d'invention. Autrement dit, pour être fidèle à une collection, il ne s'agit pas seulement de suivre ce qu'elle donne à voir de façon évidente, mais de comprendre comment elle a été produite : ses conditions d'émergence, sa temporalité, ses soutiens et ses résistances. Concernant le projet Collecta, il s'agissait de se familiariser avec la logique classificatoire mise au point par Fr.-R. de Gaignières au XVII ${ }^{\mathrm{e}}$ siècle, de comprendre ce qui avait motivé ses choix d'archivage, ainsi que les formes qu'il avait adoptées afin de pouvoir ensuite trouver une correspondance la plus adaptée et pertinente possible. C'est l'ensemble du travail historique mené de façon remarquable par A. Ritz-Guilbert (2016) qui a permis de mettre au jour cette logique interne sur laquelle le projet de design s'est ensuite appuyé, comme le principe des copies qui est au " cœur de l'entreprise savante ${ }^{15}$ » que Gaignières met en place pour permettre ses classements thématiques. 


\section{«Adaptation simplifiante » et « adaptation qui traduit"}

Concernant l'adaptation cinématographique, A. Bazin opère en particulier une distinction entre "s'inspirer d'un livre », "l'adapter au cinéma " et " le traduire au cinéma ». Pour lui l'adaptation, la mauvaise, est simplificatrice. Bien qu'il dise qu'une mauvaise adaptation n'est pas dommageable en elle-même pour la littérature, il plaide plutôt, dans le cas du cinéma, en faveur de l'idée de traduction. Pour éclairer son propos, il donne l'exemple d'une mauvaise adaptation hollywoodienne de Madame Bovary, suivant scrupuleusement le fil de l'histoire mais passant finalement à côté de l'œuvre littéraire de Flaubert. En procédant ainsi, l'industrie cinématographique nivelle l'œuvre littéraire en la réduisant à un synopsis et la diffuse de façon massive avec les moyens médiatiques dont elle dispose auprès du public. Une autre approche est selon lui possible impliquant un travail de "traduction ". Celui-ci a lieu lorsque "grâce à quelque heureux concours de circonstances, le cinéaste peut se proposer de traiter le livre autrement qu'un scénario de série, c'est un peu comme si le cinéma tout entier s'élevait au rang de la littérature " (Bazin 1987, 94). A. Bazin opère finalement une distinction entre deux types d'adaptation : une adaptation simplifiante, qui se contente, à l'instar d'imagiers, de transposer à l'identique un scénario, des personnages, un contexte, et où l'histoire sert de caution au film ; et une " adaptation qui traduit ", qui opère une traduction de l'œuvre littéraire avec les possibilités techniques du cinéma. Dans le champ des humanités numériques, un même rapport à l'adaptation peut être relevé. Dans le cas du projet Collecta, il s'agissait précisément de se rapprocher d'une adaptation traduisante. L'idée n'était pas seulement de mettre en ligne une archive mais d'inventer quelque chose : de faire exister cette collection à travers un nouveau média, l'informatique, différent des portefeuilles consultables. De la même manière qu'au cinéma il est impossible de transposer à l'identique une œuvre littéraire, il était impossible de proposer une interface qui soit l'exacte réplique de la collection, à commencer par la matérialité même de son support, sa dimension physique et tactile. Il fallait donc accepter que l'objet que nous allions créer puisse être différent de son objet référent, de façon à assumer l'identité spécifiquement numérique de l'objet à créer. 


\section{Le cas de Collecta : par-delà l'imitation}

En ce sens, il était important d'éviter l'écueil d'une transposition à l'identique, ce que Claudie Voisenat a appelé lors du colloque que nous avions organisé en 2016 " la tentation de la mimésis ", en référence à Jean-Marie Schaeffer. Nous avons donc pris quelques libertés à l'égard de la source, non pas avec la structure même de la collection, car là nous avons été des plus rigoureux, mais avec la forme et les usages qu'elle allait désormais prendre. L'idée était que le site puisse proposer une expérience différente de la consultation physique des documents, qu'il permette d'opérer des croisements inattendus, qu'il intègre des informations nouvelles comme les différents usages de la collection depuis le $\mathrm{XVII}^{\mathrm{e}}$ siècle jusqu'à aujourd'hui, qu'il participe à l'émergence de nouveaux questionnements et de nouvelles hypothèses pour les chercheurs. Élément central du projet, le modèle par points (figure 5), qui constitue une modalité d'affichage et de présentation des données inédites, permet d'embrasser d'un seul regard ${ }^{16}$ l'ensemble de l'organisation de l'inventaire de 1711 et de visualiser l'emplacement des documents recherchés dans la collection (des points se colorent en rouge en fonction des résultats de la requête).

FIGURE 5. PAGE D'ACCUEIL DU SITE WEB

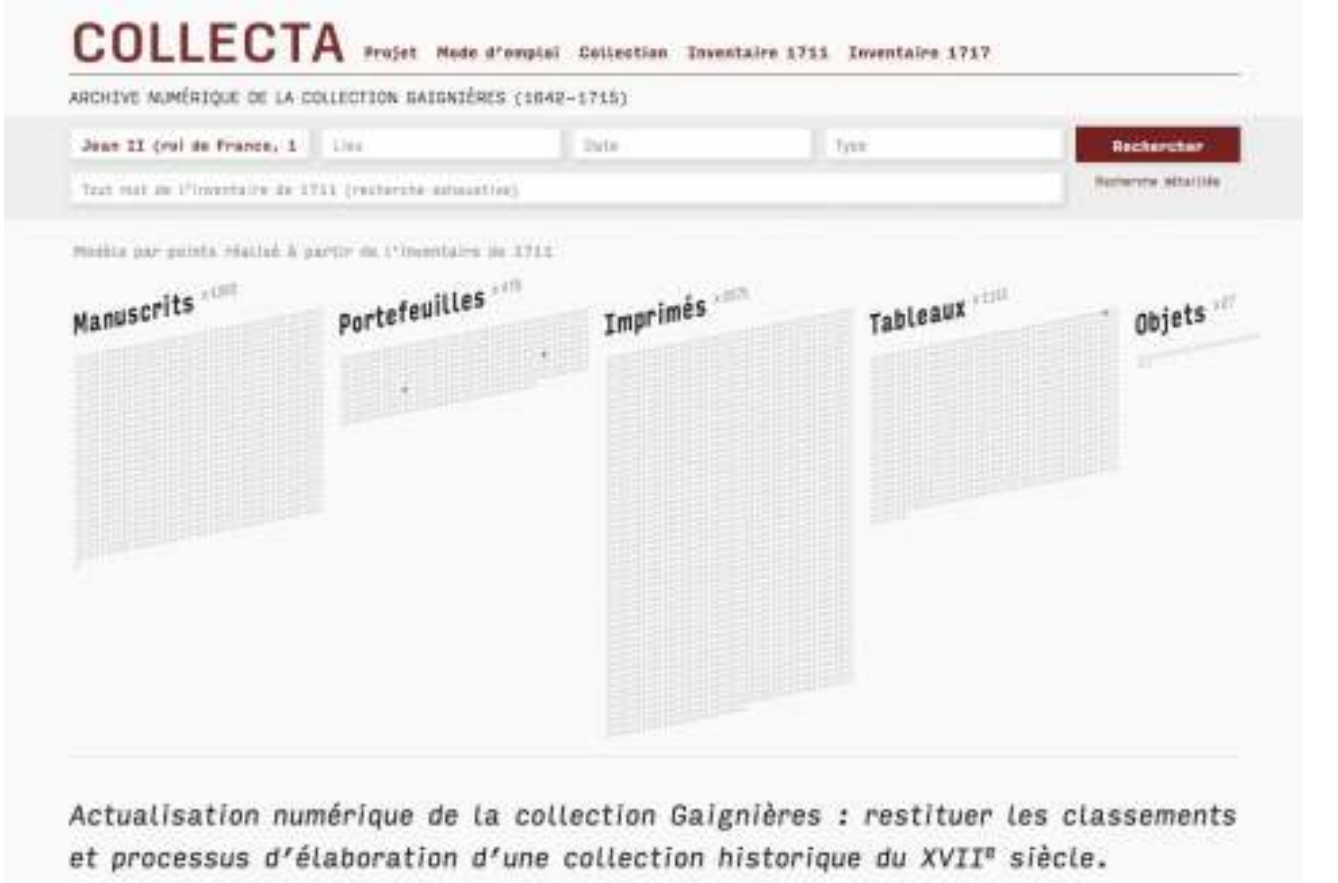

Page d'accueil du site Web de Collecta. Visualisation du modèle par points permettant de repérer l'emplacement d'un ou de plusieurs documents dans la collection, correspondant ici à Jean le Bon (roi de France). Trois points rouges cliquables s'affichent : deux dans les portefeuilles et un dans les tableaux. Le modèle par points constitue l'une des 5 entrées possibles dans la collection.

Programme de recherche Collecta, site Web : https://www.collecta.fr

Il schématise cette dernière de sorte à en devenir un accès possible, aléatoire par rapport aux recherches classiques par champs et mots-clés. Les portefeuilles et les objets sont ici matérialisés par de petits carrés 
cliquables permettant d'accéder aux fiches détaillées correspondantes. Comme l'affirme très clairement $\mathrm{A}$. Bazin à propos de l'adaptation cinématographique : "En vérité, la culture en général et la littérature en particulier n'ont rien à perdre dans l'aventure ! " (1987, 94.) De la même manière, l'histoire de l'art n'a rien à perdre dans un projet en humanités numériques, d'une part parce que la création d'une base de données et d'une interface n'altère en rien la source, elle ne lui fait pas de tort, elle ne la modifie pas directement ; d'autre part, parce qu'elle peut permettre de la compléter, d'élargir son accessibilité en favorisant la consultation de collections publiques, et d'encourager le croisement des données. En cela Collecta partage " la conviction que les dispositifs éditoriaux et les technologies numériques sont susceptibles d'ouvrir, dans certaines conditions, des possibilités inédites dans la construction d'espaces de connaissances partagées dans l'espace public » (Juanals et Minel 2017). Ce sont donc ces conditions d'ouverture d'une collection historique qui ont fait l'objet d'une attention particulière. En fait, ce que l'adaptation numérique modifie avant tout, c'est l'expérience de cette collection (sa perception et ses modalités de consultation et de réception) ainsi que son intelligibilité (le fait notamment de pouvoir en proposer une représentation). Il était donc question de procéder à une sorte de transfert, impliquant la " traduction " des portefeuilles, peintures et objets vers le numérique et le Web en vue " d'une mise à disposition de ressources patrimoniales dans le format numérique " (Juanals et Minel 2017). En dehors de la numérisation des documents (qui a été en grande partie faite par la BNF), il a fallu procéder à l'encodage des données et à l'ajout d'informations complémentaires, à la transposition numérique des inventaires, à la création des liens hypertexte, à l'automatisation de certaines procédures, à l'intégration des données graphiques (front office), ainsi qu'au renseignement progressif (bien qu'encore partiel) de la base de données (au sein d'un back office [figure 6]).

FIGURE 6. INTERFACE D'ADMINISTRATION DU SITE WEB

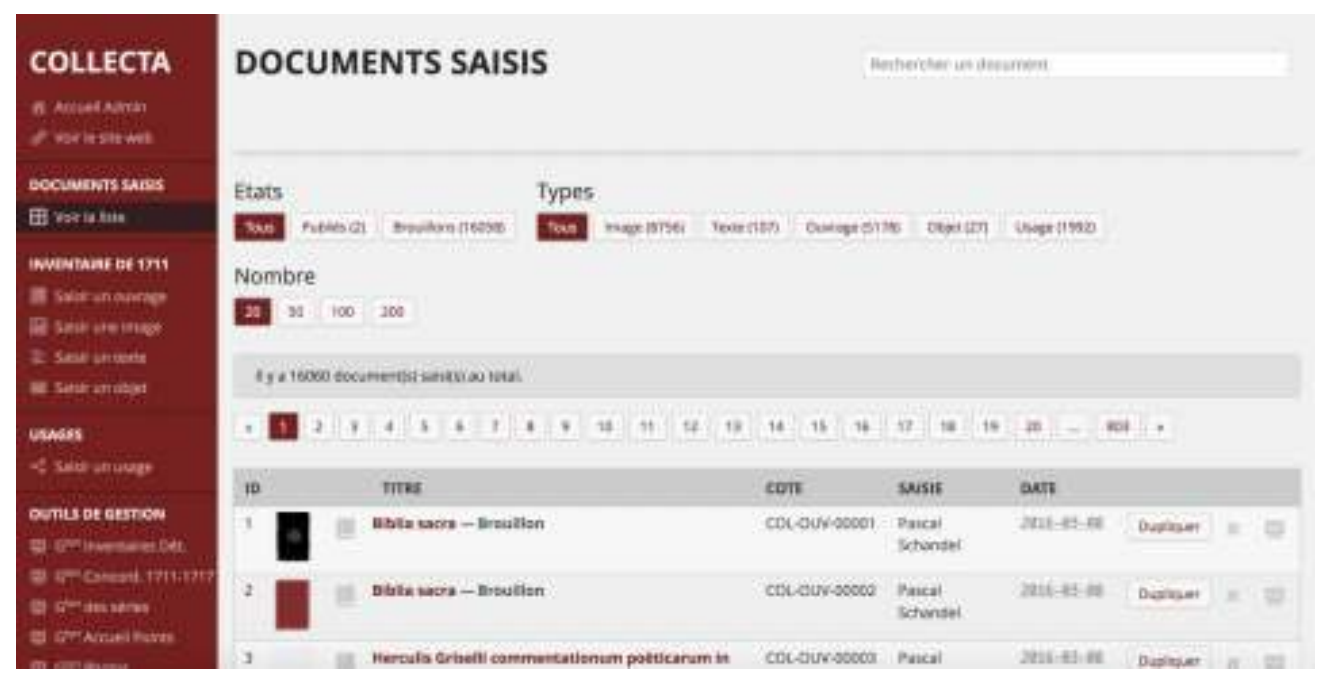

Le back office est l'espace de saisie permettant aux chercheurs de renseigner les documents. Cette interface constitue un outil de gestion des données accessible en mode administrateur.

Programme de recherche Collecta, site Web : https://www.collecta.fr. 
Ces différentes opérations ont ainsi contribué à faire passer la collection Gaignières d'une réalité de papier à une autre réalité, numérique cette fois, consultable en ligne et interopérable avec d'autres bases de données comme Gallica. Il se trouve que la collection et son organisation interne se prêtaient plutôt bien à la logique informatique et à la création d'hyperliens. Nous avons même été surpris par la proximité de la logique organisationnelle mise au point par Gaignières avec la logique informatique, procédant à des relevés et à des copies permettant à un document d'appartenir à plusieurs portefeuilles, constituant des listes, des indexations, hiérarchisant l'information par des changements de graisse et de corps typographiques dans les textes, rajoutant des signes de renvoi qui sont autant de liens vers d'autres documents. Sans aucun doute des «similitudes irrécusables " (Bazin 1987, 89) ont ainsi permis de réaliser ce passage entre le système de classement inédit de Gaignières et celui rendu possible par le numérique. Il s'agissait aussi à travers ce projet de faire exister l'esprit de la collection en faisant d'elle une "structure ouverte " et de pallier une consultation isolée des documents : "Cette structure ouverte, conçue pour être sans cesse enrichie par ajout et intercalation, a disparu de la conscience des chercheurs aujourd'hui confrontés au document isolé. Dans la collection Gaignières le document, même précis, détaillé, légendé, etc., n'est pas conçu ni utilisé pour une fin unique ou préconçue. Cette propriété de la collection la "prédispose" à un usage numérique, à une réinterprétation continue et inépuisable ${ }^{17}$. " Malgré tout, des différences importantes persistent, concernant principalement l'expérience sensible des documents, la matérialité des objets et de l'interface, ainsi que les modalités de visualisation et de consultation des documents. Il aurait d'ailleurs été dommage de les ignorer, de les atténuer ou de les occulter. Si, dans certains cas, des équivalences ont pu être établies assez facilement, dans d'autres, elles n'avaient rien d'évident et certaines n'ont pu être établies. Traduire la matérialité des objets, par exemple, s'est avéré plus complexe qu'il n'y paraissait. Ne souhaitant pas tomber dans le pastiche ou le faux-semblant, la solution trouvée, dans ce cas précis, a été de filmer la consultation de deux portefeuilles et de les présenter en vis à vis afin de donner une idée de leur matérialité et du système de correspondance platestombes/modes (figure 7). 
Matérialité des objets

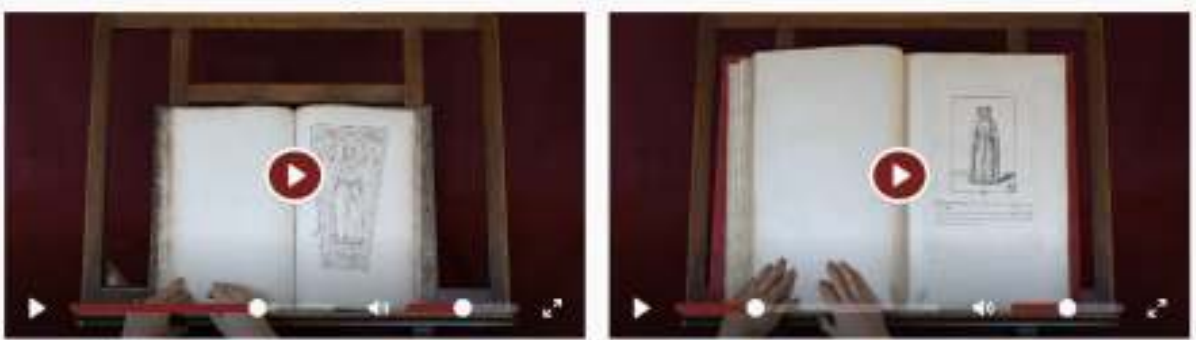

Ou tembeau (BnF, Manuscrits, tatin 5472) au cesturse (BnF, Estampes, riserve 8A-16-FOC) ; sources at interprétations

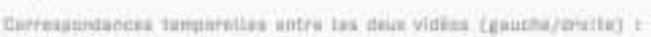

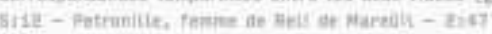

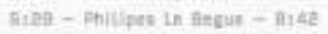

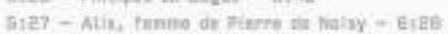

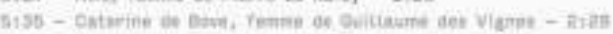

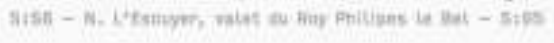

Une double vidéo comparative, mettant en parallèle le relevé graphique d'une plate-tombe (petits portefeuilles, Manuscrits, BNF), à gauche, et son interprétation dessinée classée dans un recueil de Modes (grands portefeuilles, Estampes, BNF), à droite, permet de visualiser la matérialité des objets et d'apprécier leur échelle.

Programme de recherche Collecta, site Web : https://www.collecta.fr

Si l'affichage écran peut constituer une forme de renoncement à la matérialité de la collection, en réalité, une autre tactilité s'est mise en place avec la version numérique, impliquant l'usage de l'objet informatique. Ce renoncement est d'ailleurs peut-être ce qui a nourri le processus de transfert conduisant à nous intéresser davantage à l'esprit de la collection (autrement dit au projet mis au point par Gaignières et à sa logique classificatoire) qu'à la reconstitution de sa matérialité. Ce processus d'adaptation fait exister autrement cette collection, conduit à élargir sa diffusion et à favoriser une expérience que nous dirons différenciée plutôt qu'augmentée (Sadin 2013). De même que le projet de reproduction d'un territoire à travers une carte à l'échelle 1:1 s'avère infructueux, touchant à l'absurde dans la nouvelle de Borges ${ }^{18}$, l'idée de la reproduction à l'identique d'une collection de papier n'aurait pas eu beaucoup de sens. L'adaptation est en somme la chance des humanités numériques, la possibilité même de ne pas figer la source, d'autoriser un autre mode d'existence. 


\section{Identifier les « moyens propres » du numérique}

Dès lors comment s'éloigner de la source sans la trahir ? Quel écart et degré d'éloignement accepter à l'égard de la source sans la dénaturer ? A. Bazin peut une fois encore apporter un élément de réponse, en indiquant, à propos de l'adaptation cinématographique, qu'il convient d'identifier les «moyens propres » de la technique employée, de prendre en considération la spécificité de la technique adaptante. Dans le cas du cinématographe, ils concernent aussi bien ce qui touche au progrès technique : éclairage artificiel, émulsion panchromatique, travelling, son, zoom, etc., que ses moyens d'expression : gros plan, montage, montage parallèle, montage rapide, ellipse, plans-séquences, etc. Comme il l'indique à propos de la littérature : "Le roman a ses moyens propres, sa matière est le langage, non l'image, son action confidentielle sur le lecteur isolé n'est pas la même que celle du film sur la foule des salles obscures ${ }^{19}$."

Dans le cas des humanités numériques, les " moyens propres ", ou disons plutôt les modalités spécifiques du numérique et du Web, sont notamment le code qui se décline en plusieurs langages (HTML, XHTML, XML, Java, JavaScript, PHP, etc.), les formats d'enregistrements spécifiques (comme le JPEG, GIF, PNG-8, PNG-24, SVG, BMP), les liens hypertexte, l'automatisation des procédures, le calcul via des algorithmes, le référencement par listes, la mise en réseau de l'information, l'interopérabilité. Sa réception, si elle peut être collective et partagée, est cependant la plupart du temps individuelle, elle s'opère non pas dans une salle obscure devant un grand écran comme au cinéma - mais sur un écran de moyenne dimension ou un petit écran transportable, visualisable partout et n'importe quand, pour peu qu'une connexion existe et que les appareils soient dotés d'énergie suffisante à leur fonctionnement. Le numérique opère ainsi la rencontre de la lecture confidentielle du livre et l'expérience audiovisuelle et médiatique du cinéma, à laquelle s'ajoutent la mise en réseau, l'interactivité et le partage simultané de l'information. Dans ce contexte, les designers et développeurs ont à se saisir des possibilités fonctionnelles, structurelles et esthétiques des technologies, car comme le rappelle R. T. Pédauque la neutralité de la technique n'existe pas $^{20}$. Les techniques de programmation, le choix de logiciels, de langages de programmation, le système informatique, sont autant de paramètres qui ont des conséquences sur la structure de l'information, sa mise en forme, ses modalités d'affichage, de partage, de diffusion et de consultation des contenus.

Pour Collecta, il était question à la fois d'utiliser la puissance de calcul de l'informatique et l'automatisation de certaines procédures, de faciliter de nombreuses tâches qui auraient été beaucoup trop longues à effectuer manuellement une à une, voire tout simplement impossibles à réaliser, mais aussi d'intégrer des renseignements pour lesquels les processus d'automatisation ne peuvent rien. Ajouter dans les notices des informations complémentaires (figure 8), montrer par exemple les «états et variantes " (figure 9) d'un document, ses brouillons ou ses différentes versions, accéder à des documents associés (ce qui correspond dans l’in- 
terface au bouton "Textes et ouvrages liés à ce document ») (figure 10), afficher les usages faits d'un document ultérieurement (figure 11), tout cela permet d'enrichir et de prolonger la collection Gaignières d'informations qui ne lui sont pas immédiatement liées. Ces informations complémentaires, qui échappent aux procédures automatiques de reconnaissance visuelle et textuelle, et que seul l'être humain peut fournir, sont essentielles afin d'affiner les contenus et de rendre la collection plus intelligible.

FIGURE 8. UN DOCUMENT ET SA NOTICE

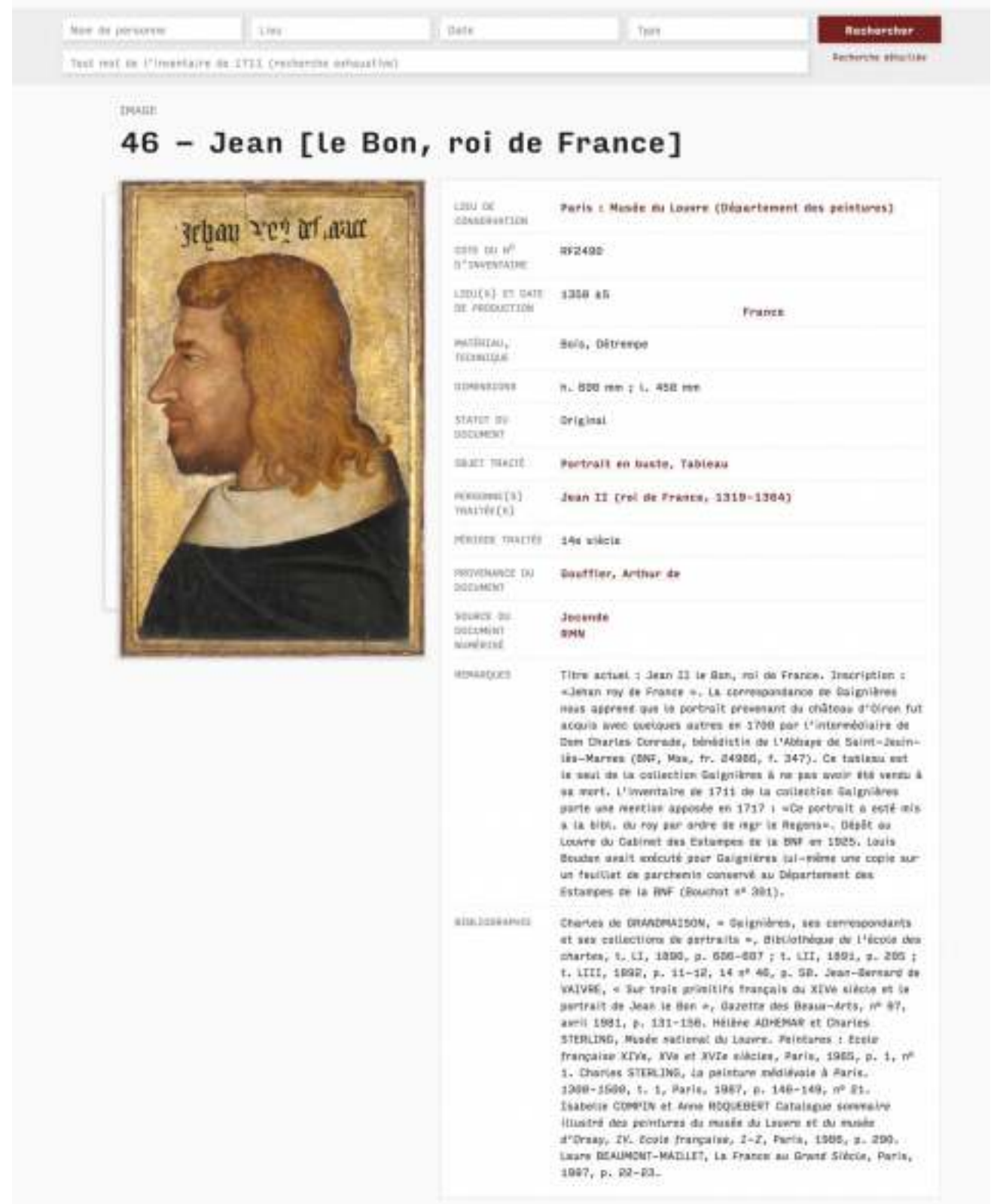

Affichage d'un document et de sa notice détaillée, indiquant la cote et les renseignements utiles, permettant d'activer les hyperliens vers les sites associés. Les champs « Remarques » et «Bibliographie » apportent des compléments d'informations connus par les historiens, renseignés dans le back office.

Programme de recherche Collecta, site Web : https://www.collecta.fr 


\section{Afficher}

\section{États et variantes de ce document}
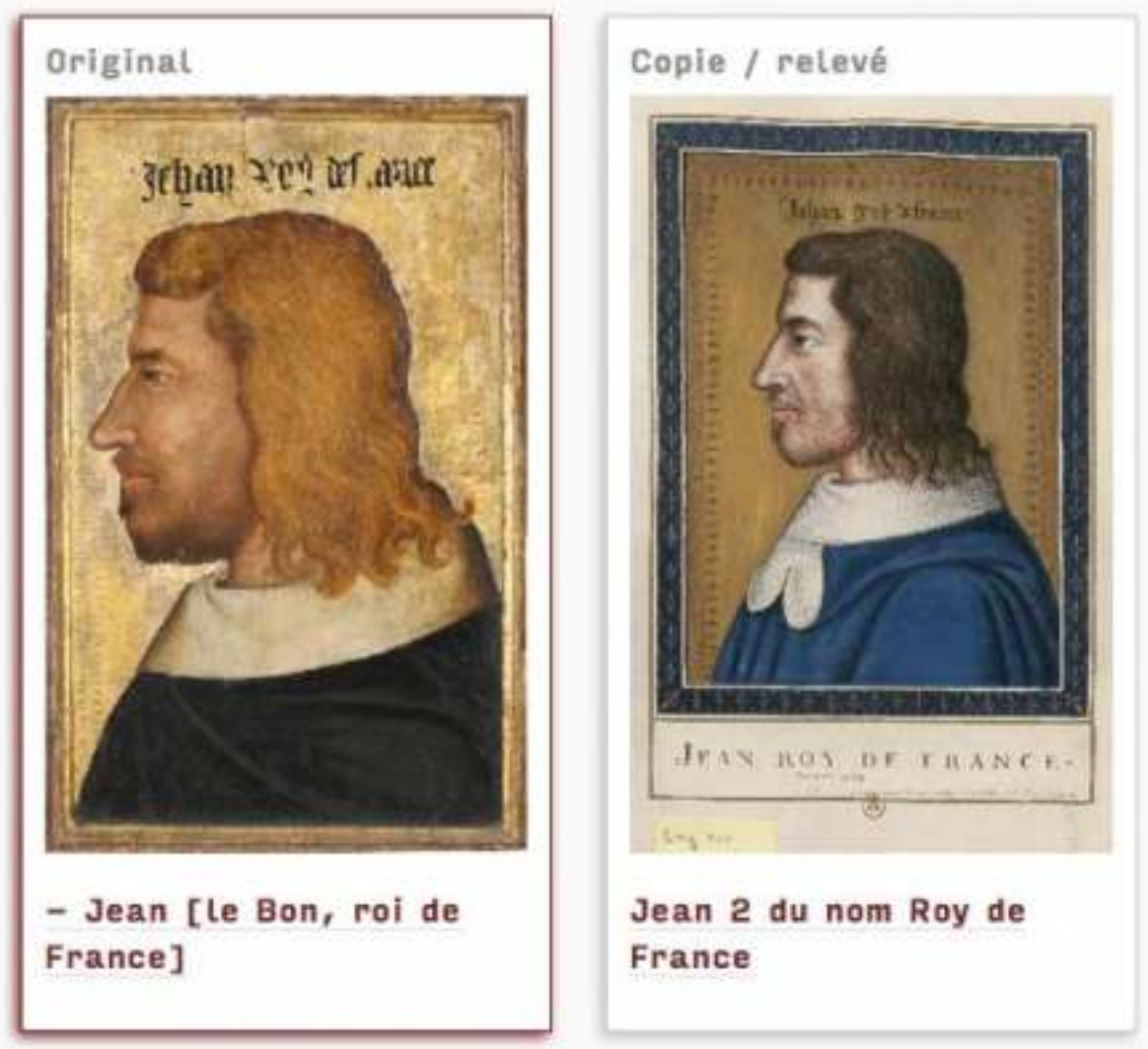

Affichage des états et variantes d'un document, ici Jean le Bon (1319-1364).

Programme de recherche Collecta, site Web : https://www.collecta.fr 


\section{Textes et ouvrages liés à ce document}

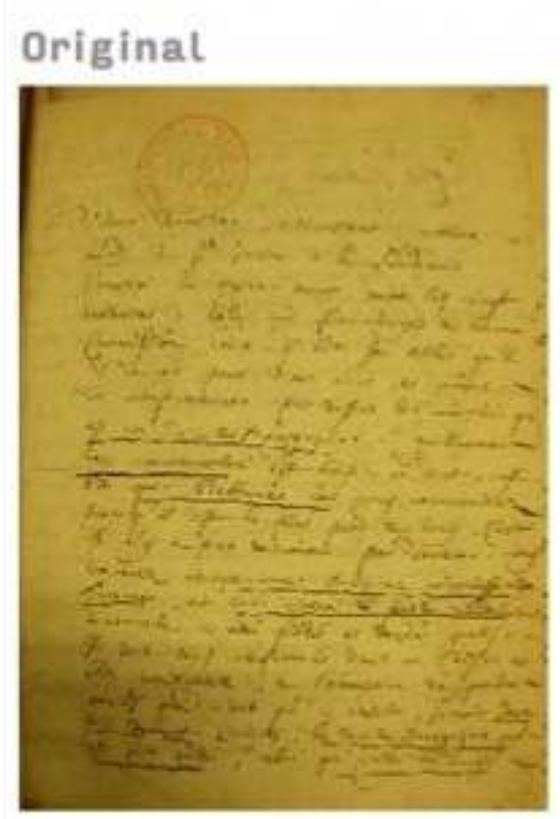

[Lettre de Dom

Charles Conrade à

Gaignières, Abbaye de

Saint-Jouin-

lès-Marnes, 15 avril

1700]

Affichage des « Textes et ouvrages liés à ce document ", ici document original en lien avec Jean le Bon (1319-1364). Il s'agit d'une lettre qui nous apprend que le portrait provenant du château d'Oiron fut acquis avec quelques autres en 1700 par l'intermédiaire de dom Charles Conrade, bénédictin de l'abbaye de Saint-Jouin-lès-Marnes.

Programme de recherche Collecta, site Web : https://www.collecta.fr 


\section{Usages de ce document}

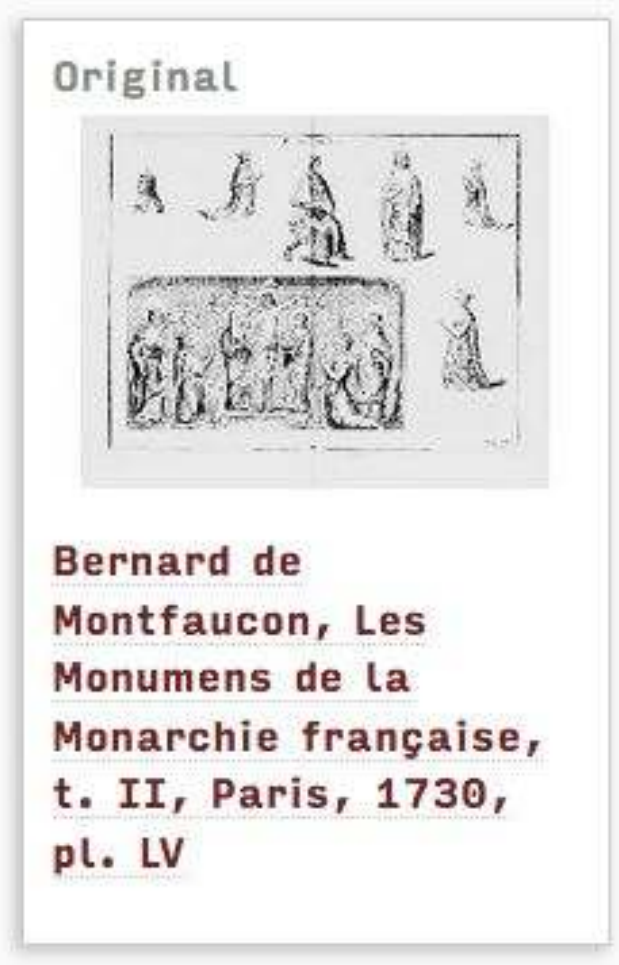

Il est également possible d'afficher, lorsqu'ils sont connus, les usages ultérieurs faits après la mort de Gaignières et la dispersion de sa collection. Ici il s'agit d'un dessin de Bernard de Montfaucon.

Programme de recherche Collecta, site Web : https://www.collecta.fr

\section{Un parallèle avec la gravure d'interprétation}

Les processus de traduction et d'adaptation ne sont pas uniquement présents au cinéma. C'est d'ailleurs A. Ritz-Guilbert qui m'a fait part d'un autre champ artistique dans lequel cette question s'est historiquement, techniquement et esthétiquement posée : la gravure d'interprétation (Préaud $2009^{21}$ ), qui repose notamment sur l'exécution de gravures d'après des peintures. Dans son travail de recherche, l'historienne de l'art Véronique Meyer analyse précisément ce phénomène, notamment le cas de la gravure de thèses, lequel s'appuie bien souvent sur l'interprétation de modèles existants (peintures ou gravures), en vue de les adapter à leur nouvelle destination et fonction.

La gravure d'interprétation, particulièrement en vogue au XVII ${ }^{\mathrm{e}}$ siècle, consiste à opérer le passage de la peinture vers l'estampe avec les moyens de la gravure. Dans le cas plus particulier de la gravure de thèses, le graveur ne se contente pas de reproduire une peinture, mais déploie un travail spécifique, qui implique une part d'invention, condui- 
sant à toutes sortes de transformations : montages, réemploi de cuivres existants, recomposition, adaptation de la gravure au nouveau format de l'image (changement parfois d'orientation du support passant de la verticale à l'horizontale), modifications des sujets, ajouts ou suppressions d'éléments de représentation, " copies partielles soit d'un groupe homogène soit de quelques figures éparses " (Meyer 1990), etc. Ces modifications qui ne sont pas toujours vertueuses dans le cas de la gravure d'interprétation, puisqu'elles sont parfois à la limite de la fraude (les modifications permettant d'échapper au soupçon possible de copie), ou comme moyen de diffusion d'une œuvre pour en augmenter sa valeur et "faciliter la vente ${ }^{22}$ » auprès des collectionneurs, pouvaient être plus ou moins importantes en fonction de leur destination et des besoins de leurs commanditaires. Dans ce passage d'une technique à une autre des différences apparaissent. Un écart se fait alors ressentir avec l'objetsource, bien souvent accentué dans le cas des gravures de thèses. Tandis que la composition est souvent dans son ensemble respectée, des différences apparaissent dès lors que le graveur opère des choix techniques, définit la nature de ses procédés graphiques (variation dans les hachures, sensibilité des tracés, etc.) et met au point le maniement personnel d'outils (façons d'encrer et d'essuyer la plaque par exemple), ce qui, de fait, permet au graveur d'apporter dans ce processus d'interprétation quelque chose qui lui est propre. Autre point commun avec le cinéma et les humanités numériques, la gravure constitue, notamment au XVII ${ }^{\mathrm{e}}$ siècle, un fantastique moyen de diffusion des images.

Cette typologie, la " gravure d'interprétation " ou l'" estampe d'interprétation ", que mentionne Maxime Préaud dans son article "Les arts de l'estampe en France au XVII ${ }^{\mathrm{e}}$ siècle : panorama sur trente ans de recherches " (Préaud 2014), se caractérise à la fois par une conformité à l'égard de l'œuvre picturale qui en constitue le modèle, et par une sorte de distanciation et d'écart à l'égard de la source. En ce sens, la gravure d'interprétation n'est pas la réplique exacte d'une œuvre picturale, plutôt une traduction possible, engageant les moyens de la gravure. V. Meyer, qu'il cite, fait d'ailleurs une distinction entre trois figures différentes : les créateurs, les interprètes et les copistes. Dans le cas de l'adaptation numérique impliquant la création d'un site Web dédié à une collection, pourrait-on adopter les mêmes distinctions ? La position de l'interprète serait sans doute la plus juste, dans la mesure où le travail réalisé part d'un existant (une collection par exemple) et que le designer ne se contente pas d'imiter un modèle existant, mais opère des choix quant aux programmes et langages de programmation utilisés, réglant des paramétrages au regard des formats, des techniques de visualisation et d'affichage. En outre, la gravure d'interprétation a longtemps souffert d'une forme de discrédit, n'étant pas toujours appréciée à sa juste valeur, apparaissant toujours comme une version secondaire (et donc mineure), à l'ombre de l'œuvre-source (et donc majeure). M. Préaud le rappelle : «La gravure d'interprétation est [...] d'ailleurs presque toujours appelée, à tort et malgré les efforts répétés de ceux qui s'y intéressent, gravure de reproduction" (Préaud 2014). Or, ainsi que le souligne V. Meyer, c'est également " la part de l'invention ", dont font preuve certains graveurs, que l'on peut reconnaître ${ }^{23}$. À l'instar de la gravure d'interprétation, les interfaces numériques dédiées à des collections historiques pourraient bel et bien pâtir d'un même dénigrement, pouvant apparaître comme 
des reproductions imparfaites et secondaires par rapport à la source, ou seulement fonctionnelles, et pour lesquelles la dimension créative serait minime, alors même qu'une part d'invention peut s'y développer.

\section{De l'impureté}

L'impureté dont parle A. Bazin à propos de l'adaptation cinématographique pourrait bien s'avérer également caractéristique des humanités numériques dès lors qu'elles opèrent la rencontre et l'hybridation de champs a priori éloignés les uns des autres, à savoir dans le cas que nous avons évoqué : l'histoire de l'art, le design et l'informatique. Il s'agit en quelque sorte d'une impureté de nature à laquelle les humanités numériques ne peuvent guère échapper, et qui, c'est ce que nous voulons démontrer ici, peut s'avérer vertueuse. À se référer aux propos du romancier et essayiste Guy Scarpetta, l'impureté pourrait caractériser un certain type d'œuvres, relevant du baroque au sens où de l'hétérogénéité est en jeu. Cette impureté, précise-t-il, n'est pas une impureté post-moderne renvoyant à des signes vides de sens. Dans notre cas, elle serait plutôt caractéristique d'un type de production qui assume sa relation à l'artifice, à la technique. Cette impureté qui consiste à déplacer des documents historiques au sein d'Internet, à les transformer en matière numérique - ce qui est quand on y pense un acte tout à fait radical -, fait évoluer les disciplines tant de l'histoire de l'art que du design. Les humanités numériques conduisent, de fait, l'histoire de l'art à se confronter à la programmation, aux codes, à l'informatique, au partage des données, à l'open source, à l'interopérabilité, à la mise en réseau de l'information, aux big data, etc. Le design est amené quant à lui à rencontrer des enjeux historiques qui ne lui sont pas toujours immédiatement familiers comme l'archive, l'histoire médiévale, les sceaux, l'héraldique, la codicologie, etc., le conduisant à élargir son champ de questionnements. Aussi, cette impureté ne serait pas en elle-même dommageable dès lors qu'elle favoriserait la rencontre de domaines hétérogènes capables de "s'éclairer réciproquement »(Scarpetta 1988, 12).

\section{Valeurs documentaires, valeurs esthétiques et valeurs d'usage}

Assurément, les auteurs de bases de données et de sites Web dédiés à une archive numérique ne suivent pas nécessairement les mêmes buts dès lors que les uns s'intéressent à l'objet créé avant tout comme ressource documentaire, et que les autres pensent en priorité l'ergonomie du site, ses modalités de navigation, ses qualités visuelles ainsi que l'expérience proposée.

Les enjeux documentaires du site Collecta, en tant que publication savante, sont multiples et l'on peut énoncer quelques points principaux qui ont motivé les historiens :

1. Restituer la logique classificatoire de Gaignières en suivant l'inventaire de 1711 et permettre la lecture de l'inventaire de 1717 avec ses différentes mains (figure 12)

2. Reconstituer les portefeuilles et l'ordre des feuillets 
3. Intégrer les usages ultérieurs de la collection en s'inscrivant dans son esprit d'ouverture sans perdre pour autant son " unité " (Lauxerois 2004)

Du côté du design, trois principales préoccupations ont guidé le travail :

1. Ne pas entrer en concurrence avec la collection, ne pas la pasticher ou vouloir la copier formellement

2. Proposer une expérience utilisateur qui soit inédite pour autant que la structure interne de la collection soit parfaitement respectée

3. Faire en sorte que la création de ce site et de cette base de données soit l'occasion d'inventer quelque chose de différent des sites existants, en phase avec son contenu

FIGURE 12. INVENTAIRE DE 1717

\section{COLLECTA}

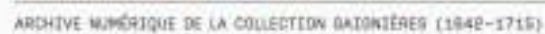

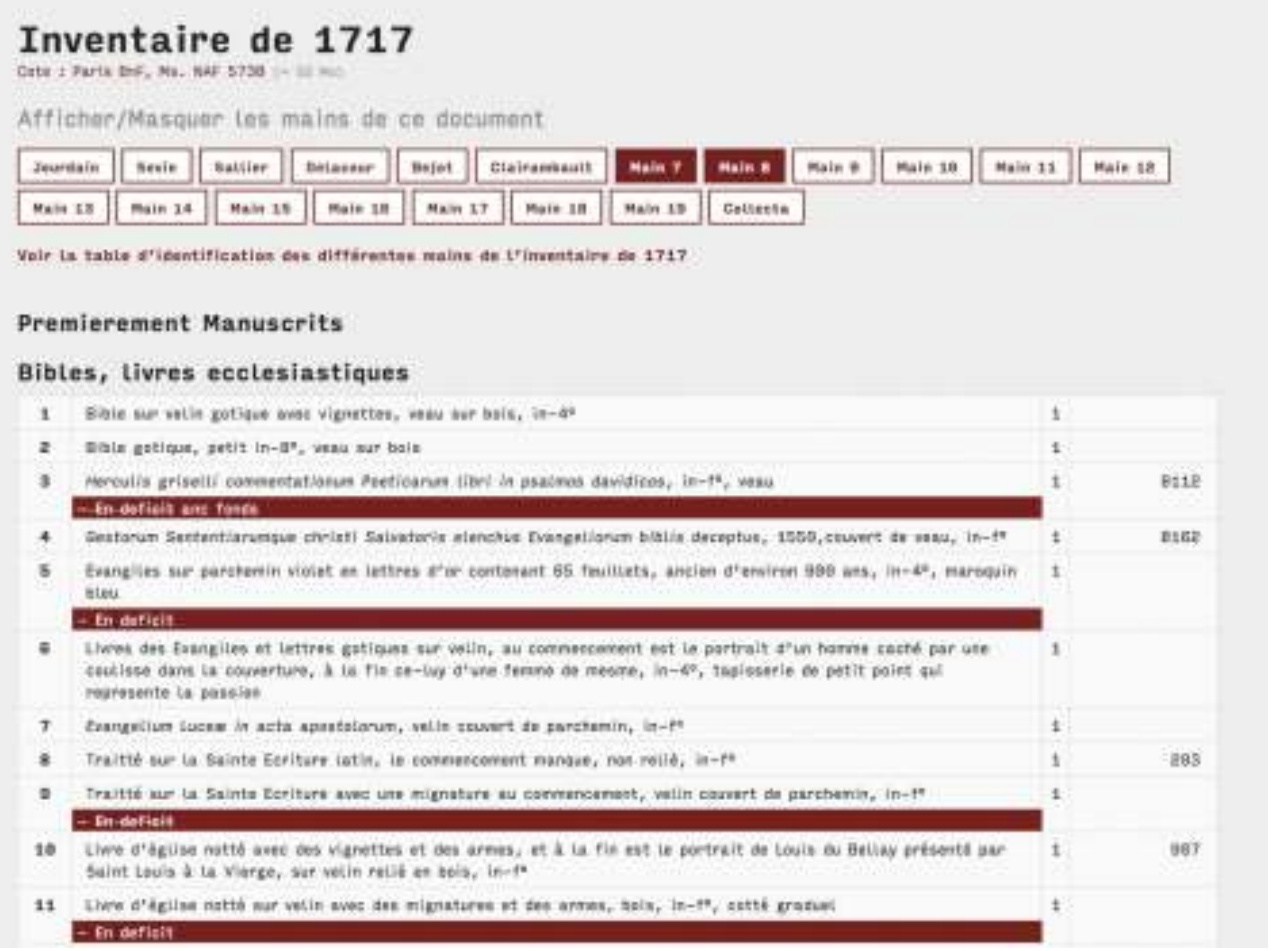

L'inventaire de 1717 permet de visualiser les auteurs (mains) qui ont contribué à l'établir. Cette synthèse consultable et visualisable n'avait jamais été réalisée auparavant.

Programme de recherche Collecta, site Web : https://www.collecta.fr

Pour répondre à ces différents objectifs, il aura fallu sortir de la «fidélité captive " (R. T. Pédauque) ou de la "fidélité comme servitude " (A. Bazin) afin d'envisager le rapport à la source comme ouvert à une part de création, évitant l'écueil de ce que Jean Lauxerois appelle la « fossilisation ", qui " contredit le principe esthétique de la métamorphose des œuvres" (Lauxerois 2004, 5-32). Il ne s'agit pas seulement de restituer la numérisation d'un document ou d'un fonds, mais de partir de cet existant pour inventer tant sur le plan de la forme que sur le plan des usages, de l'organisation des données et de la technique. Au regard de ce cahier des charges à double entrée, des orientations techniques ont pu être prises comme l'interopérabilité avec d'autres bases de données telle Gallica, le choix de l'open source et de la programmation de la base de 
données de type MySQL, sans passer par des logiciels préexistants qui auraient imposé des contraintes supplémentaires. Si des perspectives différentes sont envisageables, elles ne sont pas pour autant divergentes, car l'objet obtenu constitue un seul et même objet. L'expérience du cinéma, fort d'une histoire plus ancienne que le design, l'informatique et les humanités numériques, peut apporter un éclairage précieux quant à la façon dont il est possible de conduire un projet en humanités numériques incluant une approche par le design. "Si le cinéma est aujourd'hui capable de s'attaquer efficacement au domaine romanesque et théâtral, c'est d'abord qu'il est assez sûr de lui-même et maître de ses moyens pour s'effacer devant son objet. C'est qu'il peut enfin prétendre à la fidélité - non plus illusoire fidélité de décalcomanie - par une intelligence intime de ses propres structures esthétiques, condition préalable et nécessaire au respect des œuvres qu'il investit " (Bazin 1987, 99-101). Parce que les humanités numériques n'ont pas encore atteint une pleine maturité à l'égard de leurs moyens, il est plus que jamais nécessaire d'explorer les possibilités du numérique afin d'apprécier ce que ce dernier peut véritablement pour ces collections et ce qu'il ne peut pas, et de faire émerger d'autres moyens qui n'existent pas encore. Finalement, le pari pour les projets en humanités numériques est que le numérique et l'informatique sont tout autant l'occasion d'une lecture nouvelle des documents, d'une mise à distance inattendue d'une collection, que d'une expérience sensible inédite d'une archive et de sa possible médiation par le numérique. Malgré des sensibilités et attendus différents, l'une des leçons que l'on peut tirer de cette expérience est qu'il apparaît primordial de ne pas dissocier le fond de la forme, et que dès que l'un est oublié, il fait défaut à l'autre. Collecta, bien qu'animé en partie par une "mission de sauvetage ", n'envisage pas pour autant le numérique comme la possibilité de sauver une collection de sa " ruinification " (Bazin 1987). Il est plutôt tourné vers l'avenir et les possibilités qu'offre le numérique. La base de données, si elle semble pouvoir sauver l'archive de sa fragile existence (ce qui en réalité est loin d'être garanti étant donné l'obsolescence des technologies et la fragilité de ses supports), est surtout l'occasion de découvrir des choses jusque-là inaperçues, d'accéder différemment à l'objet qu'elle documente. Si Gaignières se heurte à l'impossible exhaustivité, il ne renonce pas pour autant à la possibilité d'accueillir de nouveaux documents. Cela constitue sans doute un point commun entre son travail et celui que nous avons voulu mener à travers ce projet : le souhait d'actualiser l'ensemble de la collection Gaignières sans la figer, tout en cherchant à la penser comme une archive encore vivante, non figée, non "stagnante ", en écho à la formule de Bruno Tackels (2012), pouvant accueillir des compléments d'information de façon illimitée, en prévoyant l'annotation des administrateurs et des historiens au sein des notices, mais aussi en rendant possible l'ajout des usages et de documents associés. Comme le souligne Bruno Bachimont (2016), « l'accumulation nie la possibilité d'une préservation active, qui est alors au mieux aveugle car ne reposant que sur la seule force de nos outils automatiques et les procédures gestionnaires ". Or, la question pour Collecta n'est pas seulement d'accumuler (Bachimont parle d'une "frénésie d'accumulation ») mais bien d'entrer en interaction avec les contenus. Pris dans la " contradiction » d'une sauvegarde de cette fantastique collection, et d'une « instabilité des techniques "(Bachimont 2016), l'actualisation que nous avons 
voulu mettre en place consiste avant tout à " atteindre une autre vérité " (Tackels 2012). Il n'est donc pas certain que Collecta sauvera la collection Gaignières de la destruction et de l'usure du temps, néanmoins, une chose est sûre, c'est que sa publication numérique permet à cette collection de sortir de l'ombre, de gagner en visibilité, et de voir ses usages et usagers se diversifier ; rejoignant la formule de Bazin : «En vérité, il n’y a point concurrence et substitution, mais adjonction d'une dimension nouvelle. " Pour le dire avec les mots de N. Katherine Hayles, ce sont " différents régimes attentionnels" (Hayles 2016) qui ici se conjuguent. $\mathrm{Au}$ terme de cette première phase de recherche, il apparaît certain, et l'expérience Collecta nous conduit à cette conclusion, qu'un projet en humanités numériques a tout intérêt à s'intéresser à son design, non parce qu'il est capable de produire des formes attrayantes, mais parce qu'il est capable de penser l'usage et les modalités de présentation d'une chose en lien avec ce qu'elle est. De la même façon qu'au cinéma le montage agit sur la forme et l'esthétique générale du film, dans le cas de la création d'une archive Web, la structuration des données et les choix techniques jouent aussi sur ses formes et les modalités de sa réception, que l'on ne peut ignorer.

\section{Bibliographie}

Bachimont, Bruno. 2017. Patrimoine et numérique : technique et politique de la mémoire. Brysur-Marne : INA Éditions.

Bazin, André. 1987 [1952]. « Pour un cinéma impur. Défense de l'adaptation ». Dans Qu'estce que le cinéma?, 81-106. Paris : Éditions du Cerf.

Berger, Pierre, Gérald Grunberg et Jean Lauxerois. 2004. La Notion de collection : ou comment lutter contre l'éparpillement des choses dans le monde. Paris : Éditions de la Bibliothèque publique d'information. http://books.openedition.org/bibpompidou/1451.

Borgès, Jorge Luis. 1951. Histoire de l'infamie, histoire de l'éternité. Paris : Le Rocher.

Hayles, N. Katherine. 2016. Lire et penser en milieux numériques : attention, récits, technogenèse. Grenoble : Ellug.

Juanals, Brigitte et Jean-Luc Minel. 2017. Enjeux numériques pour les médiations scientifiques et culturelles du passé. Nanterre : Presses universitaires de Paris Nanterre.

Le Deuff, Olivier, éd. 2014. Le Temps des humanités digitales : la mutation des sciences humaines et sociales. Limoges : Fyp.

Meyer, Véronique. 1984. "Gilles Rousselet et la gravure d'interprétation au XVII ${ }^{\mathrm{e}}$ siècle ". Thèse de doctorat, université Paris I-Sorbonne.

Meyer, Véronique. 1990. "Copies et montages dans la gravure d'allégories ». Nouvelles de l'estampe, 5-12. https://hal.archives-ouvertes.fr/hal-01919742v1.

Pédauque, Roger T. 2006. Le Document à la lumière du numérique. Caen : C\&F éditions.

Préaud, Maxime. 2014. " Les arts de l'estampe en France au XviI ${ }^{\mathrm{e}}$ siècle : panorama sur trente ans de recherches ». Perspective, août. http://perspective.revues.org/1308.

Ritz-Guilbert, Anne. 2016. La Collection Gaignières. Un inventaire du royaume au XVII ${ }^{e}$ siècle. Paris : CNRS Éditions.

Ritz-Guilbert, Anne et Sophie Fétro, éd. 2016. Collecta. Des pratiques antiquaires aux humanités numériques. Paris : École du Louvre.

Sadin, Éric. 2013. L'Humanité augmentée. L'administration numérique du monde. Montreuil : L'Échappée.

Scarpetta, Guy. 1988. L’Artifice. Paris : Grasset \& Fasquelle.

Tackels, Bruno. 2012. «Walter Benjamin, lecteur absolu ». Revue de la BNF 41 (2) : 5-10. http s://www.cairn.info/revue-de-la-bibliotheque-nationale-de-france-2012-2-page-5.htm. 


\section{Notes}

1 En plus des porteuses de projet, Anne Ritz-Guilbert et Sophie Fétro, ce sont : Pascal Schandel (historien de l'art, chargé de mission, École du Louvre), Anthony Masure (designer d'interfaces, chargé de mission, université Paris 1 Panthéon-Sorbonne), Matthieu Lacroix (développeur-programmeur), Josselin Morvan (encodeur TEI), Virginia Cassola (doctorante, École du Louvre), Camille Jolin (élève en master 2, École du Louvre), AnneSophie Rincel (élève en master 2, École du Louvre) et Stéphane Lab (élève en master 2, École du Louvre).

2 https://www.collecta.fr.

3 https://www.collecta.fr/contenu-collection.php.

4 https://www.collecta.fr/contenu-collection.php.

5 https://www.collecta.fr/contenu-projet-collecta.php.

6 https://www.collecta.fr/index.php.

7 http://enccre.academie-sciences.fr/encyclopedie/.

8 https://arachne.hypotheses.org.

9 http://passes-present.eu/fr/guides-de-paris-les-historiens-des-arts-et-les-corpus-nu meriques-363.

10 https://cahier.hypotheses.org/e-stampages.

11 https://medialab.sciencespo.fr/activites/the-experiments-in-art-and-technology-eat-d atascape/.

12 Pédauque (2006, 116, chap. « Pour une élucidation de la transformation documentaire. La notion d'invariant»).

13 « [S]i la copie en cascade (comme dans le cas de la photocopie) peut nuire à la qualité du document ainsi reproduit, la nature du double produit numériquement est différente, de même la numérisation peut nuire à l'invariance d'un document ainsi reproduit " (Pédauque 2006).

14 " Aujourd'hui, le chercheur consulte des pièces de Gaignières dans les fonds français, latin, italien, anglais, allemand, arabe, Clairambault, dans les "pièces originales" du département des Manuscrits, dans les portefeuilles de dessins au département des Estampes et de la Photographie et à la bibliothèque Bodléienne d'Oxford. "

15 https://www.collecta.fr/contenu-projet-collecta.php.

16 «Le modèle par points répond au désir de Gaignières d'appréhender la collection visuellement "pour trouver tout d'un coup toutes les matières qu'il contenoit et dont la multitude était immense" " (Ritz-Guilbert et Fétro 2016, 27).

17 https://www.collecta.fr/contenu-projet-collecta.php.

18 « En cet empire, l'Art de la Cartographie fut poussé à une telle Perfection que la Carte d'une seule Province occupait toute une Ville et la Carte de l'Empire toute une Province. Avec le temps, ces Cartes Démesurées cessèrent de donner satisfaction et les Collèges de Cartographes levèrent une Carte de l'Empire, qui avait le Format de l'Empire et qui coïncidait avec lui, point par point. Moins passionnées pour l'Étude de la Cartographie, les Générations Suivantes réfléchirent que cette Carte Dilatée était inutile et, non sans impiété, elles l'abandonnèrent à l'Inclémence du Soleil et des Hivers. Dans les Déserts de l'Ouest, subsistent des Ruines très abîmées de la Carte. Des Animaux et des Mendiants les habitent. Dans tout le Pays, il n'y a plus d'autre trace des Disciplines Géographiques. » (Suárez Miranda, Viajes de Varones Prudentes, libro cuarto, cap. XLV, Lérida, 1658, cité par Borges [1951, 129-130]).

19 Bazin (1987, 95).

20 Pédauque (2006, chap. «La non neutralité de la technique »).

21 Voir également la thèse de doctorat de Véronique Meyer (1984).

22 Meyer (1990).

23 « Nous avons également tenté de mettre en évidence la liberté prise par quelques graveurs face à leur modèle, c'est-à-dire la part de l'invention dans certaines copies » (Meyer 1990). 


\section{Auteur}

\section{Sophie Fétro}

EA 7539 Institut Acte, université Panthéon-Sorbonne, Paris, France

Sophie Fétro est maître de conférences à l'université Paris 1 Panthéon-Sorbonne, designer et chercheuse, spécialisée dans l'étude du design et les pratiques numériques d'assistance au projet, elle a travaillé sur le programme de recherche « La fin des cartes, territoires rêvés, territoires normalisés "(2013-2015), a été membre du comité scientifique du programme de recherche " Nouvelles histoires du faire : architecture et design à l'ère de la fabrication digitale " (Labex CAP, porté par le Centre Pompidou, sous la direction de Marie-Ange Brayer), et a été co-responsable avec Anne Ritz-Guilbert du programme de recherche "Collecta. Archive numérique de la collection Gaignières (1642-1715) ", participant à la conception, au prototypage et à la réalisation du site Web Collecta.

Sophie.fetro@univ-paris1.fr

\section{Droits d'auteur}

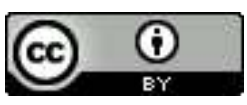

Les contenus de la revue Humanités numériques sont mis à disposition selon les termes de la Licence Creative Commons Attribution 4.0 International. 